\title{
COVERING LEMMAS AND THE SHARP FUNCTION
}

\author{
RICHARD J. BAGBY AND DOUGLAS S. KURTZ
}

\begin{abstract}
We present some new measure-theoretic inequalities for families of cubes covering open sets in $\mathbf{R}^{n}$ and use these inequalities to estimate the Hardy-Littlewood maximal function $M f$ in terms of the sharp function $f^{\#}$.
\end{abstract}

1. Introduction. We develop a simple but powerful covering lemma with applications to harmonic analysis in $\mathbf{R}^{n}$. In its simplest form the lemma states that an open set $\Omega$ of finite measure can be covered by a sequence of cubes having total measure comparable to that of $\Omega$ in such a way that each cube is surrounded by the complement of $\Omega$. That is, for each cube $Q$ in the covering, every larger cube meeting $Q$ also meets $\Omega^{c}$. While the standard decomposition of open sets on the line has this property, previously known substitutes in higher dimensions do not. This property makes our covering lemma useful for analyzing the level sets of maximal functions associated with the family of all cubes in $\mathbf{R}^{n}$.

We use our covering lemma to bound the Hardy-Littlewood maximal function $M f$ in terms of the "sharp function" $f$ of Fefferman and Stein [5]. We prove

$$
(M f)_{w}^{*}(t) \leqslant C\left(f^{\sharp}\right)_{w}^{*}(2 t)+(M f)_{w}^{*}(2 t),
$$

where $g_{w}^{*}$ denotes the decreasing rearrangement of $g$ with respect to the weighted measure $w(x) d x$. Thanks to our covering lemma, our derivation of (1.1) is both simple and enlightening.

Just as we observed in [1], inequality (1.1) implies best possible versions of the previously known estimates relating $M f$ and $f^{\#}$. It is an improvement of the estimate first proved by Fefferman and Stein, who compared distribution functions with respect to Lebesgue measure instead of rearrangements with respect to weighted measures. While their arguments can be extended to the weighted case, their basic inequality is simply not as strong as (1.1). Bennett and Sharpley [3] proved an unweighted version of (1.1) and developed most of its consequences, but their proof does not extend to the weighted case. Thus, both the content and our proof of (1.1) are of interest.

In $\$ 2$ we present background material and definitions. The covering lemmas are proved in \$3. The last section contains the rearrangement inequalities and their consequences.

Received by the editors March 13, 1984.

1980 Mathematics Subject Classification. Primary 42B25, 46E30.

Key words and phrases. $A_{\infty}$ weight function, covering lemma, Hardy-Littlewood maximal function, sharp function. 
2. Preliminaries. Points in Euclidean space $\mathbf{R}^{n}$ are denoted simply as $x$ or $y$. We shall always assume that $f$ is a Lebesgue measurable, locally integrable, complex-valued function on $\mathbf{R}^{n}$. For $f$ integrable, we denote its Lebesgue integral as $\int f(x) d x$. The Lebesgue measure of a set $E$ is denoted $|E|$. We use the letter $Q$ or the word cube to refer to any translate of a set $[0, s]^{n}, 0<s<\infty$.

We define the Hardy-Littlewood maximal function $M f$ and the sharp function $f^{\#}$ by

$$
M f(x)=\sup \frac{1}{|Q|} \int_{Q}|f(y)| d y
$$

and

$$
f^{\sharp}(x)=\sup \frac{1}{|Q|} \int_{Q}\left|f(y)-f_{Q}\right| d y,
$$

where the suprema are taken over all cubes containing $x$, and $f_{Q}$ is the mean value $(1 /|Q|) \int_{Q} f(y) d y$.

Throughout our paper, $w$ is a fixed weight function in the class $A_{\infty}$ of Muckenhoupt [4]. That is, there are constants $A, p \in(1, \infty)$ such that

$$
\int_{(M f>\lambda)} w(x) d x \leqslant A \lambda^{-p} \int|f(x)|^{p} w(x) d x
$$

for all measurable functions $f$ and all $\lambda>0$. We shall ignore the trivial cases where $w$ vanishes identically or is identically infinite; (2.1) then implies that $w$ is positive almost everywhere.

We identify $w$ with the absolutely continuous measure having density function $w(x)$ and write $w(E)$ for $\int_{E} w(x) d x$. The weights in $A_{\infty}$ satisfy

$$
w(Q) \leqslant A(|Q| /|E|)^{p} w(E)
$$

and

$$
w(E) \leqslant C(|E| /|Q|)^{\delta} w(Q)
$$

for all $E \subset Q$. While (2.2) follows easily from (2.1), (2.3) is quite subtle. Note that the constants $C$ and $\delta$ depend only on $n, p$, and $A$. A discussion of $A_{\infty}$ weights is contained in the article by Coifman and Fefferman [4].

For $f$ a measurable function on $\mathbf{R}^{n}$, the rearrangement of $f$ with respect to $w$ is defined on $(0, \infty)$ by

$$
f_{w}^{*}(t)=\inf \{\lambda>0: w(\{x:|f(x)|>\lambda\}) \leqslant t\} .
$$

We also write

$$
f_{w}^{* *}(t)=\frac{1}{t} \int_{0}^{t} f_{w}^{*}(s) d s
$$

for the averaged rearrangement with respect to $w$.

We will generate a number of constants; these will be assumed to depend only on $n, p$, and $A$ from (2.1). 
3. Covering lemmas. Here we prove two covering lemmas for open sets in $\mathbf{R}^{n}$. The first is a weighted version of a covering lemma used by Bennett and Sharpley [3]; it is essentially a Calderon-Zygmund decomposition. The second is completely new and somewhat surprising, although an easy consequence of the first.

3.1 LeMMA. There is a constant $B$ such that each open set $\Omega$ with $w(\Omega)<\infty$ can be covered by a sequence of nonoverlapping cubes $\left\{Q_{k}\right\}$ each satisfying

(a) $w\left(Q_{k}\right) \leqslant B w\left(Q_{k} \cap \Omega\right)$, and

(b) $\left|Q_{k}\right| \leqslant 2\left|Q_{k} \cap \Omega^{c}\right|$.

We shall see that our method of choosing the cubes works for all sufficiently large constants $B$. Fixing $B$, we first cover $\mathbf{R}^{n}$ by nonoverlapping cubes such that each cube satisfies $w(Q)>B w(\Omega)$. This is easily done in the unweighted case since the Lebesgue measure of a cube depends only on its geometric size. Here we substitute the doubling property of measures defined by $A_{\infty}$ weights. By defining a grid of cubes for which the diameters grow in proportion to the distance from the origin, we ensure an arbitrarily large lower bound on the $w$-measure of the cubes.

Proof. If $Q$ and $Q^{\prime}$ are intersecting cubes of equal side length, it follows from (2.2) that there is a constant $C \geqslant 1$ so that $w(Q) \leqslant C w\left(Q^{\prime}\right)$. This implies there is an $\varepsilon>0$ so that $w(3 Q) \geqslant(1+\varepsilon) w(Q)$ and, hence, $w\left(3^{k} Q\right) \geqslant(1+\varepsilon)^{k} w(Q)$. Therefore, we can choose a cube with arbitrarily large $w$-measure.

Let $Q_{1}$ be a cube centered at the origin with $w\left(Q_{1}\right) \geqslant C B w(\Omega)$. For each $m \geqslant 1$ we partition $3^{m} Q_{1} \sim 3^{m-1} Q_{1}$ into $3^{n}-1$ congruent subcubes; each has $w$-measure at least $(1 / C) w\left(3^{m-1} Q_{1}\right)$.

Having covered $\mathbf{R}^{n}$ with nonoverlapping cubes each having $w(Q)>B w(\Omega)$, we discard those cubes which do not intersect $\Omega$. For each remaining cube $Q$, we partition $Q$ into $2^{n}$ congruent subcubes; let $\tilde{Q}$ denote any of these. By (2.2) we then have

$$
B w(\tilde{Q} \cap \Omega) \leqslant B w(Q \cap \Omega)<w(Q) \leqslant 2^{n p} A w(\tilde{Q}) .
$$

Include $\tilde{Q}$ in the covering of $\Omega$ if $w(\tilde{Q}) \leqslant B w(\tilde{Q} \cap \Omega)$; otherwise repeat the process inductively, treating $\tilde{Q}$ exactly as $Q$ was treated. As long as $B>2^{n p} A$, each point in $\Omega$ will lie in some selected cube.

Applying (2.2) to each selected cube $\tilde{Q}$, we see

$$
(|\tilde{Q} \cap \Omega| /|\tilde{Q}|)^{p} \leqslant A w(\tilde{Q} \cap \Omega) / w(\tilde{Q}) \leqslant 2^{n p} A^{2} / B
$$

The proof is completed by taking $B$ large enough that this last quantity is at most $2^{-p}$.

3.2 LEMMA. There is a constant $C$ such that each open set $\Omega$ in $\mathbf{R}^{n}$ with $w(\Omega)<\infty$ can be covered by a sequence $\left\{Q_{k}\right\}$ of nonoverlapping cubes in such a way that:

(a) $\sum w\left(Q_{k}\right) \leqslant C w(\Omega)$

(b) if $Q \cap Q_{k} \neq \varnothing$ and $\left|Q_{k}\right| \leqslant|Q|$, then $|Q| \leqslant 2\left|Q \cap \Omega^{c}\right|$.

Proof. Let $\tilde{\Omega}=\left\{x \in \mathbf{R}^{n}: M \chi_{\Omega}(x)>2^{-n-1}\right\}$. Then $\tilde{\Omega}$ is open, $\Omega \subset \tilde{\Omega}$, and $w(\tilde{\Omega})$ $\leqslant 2^{(n+1) p} A w(\Omega)$ by $(2.1)$. We apply Lemma 3.1 to $\tilde{\Omega}$. Clearly the sequence $\left\{Q_{k}\right\}$ of 
selected cubes then satisfies

$$
\sum w\left(Q_{k}\right) \leqslant \sum B w\left(Q_{k} \cap \tilde{\Omega}\right) \leqslant B w(\tilde{\Omega}) \leqslant C w(\Omega) .
$$

Suppose now that $Q$ is any cube such that $Q$ meets $Q_{k}$ and $\left|Q_{k}\right| \leqslant|Q|$. Let $\tilde{Q}$ be a cube containing both $Q$ and $Q_{k}$ and having $|\tilde{Q}|=2^{n}|Q|$. For $x \in \tilde{Q}$ we have $M \chi_{\Omega}(x) \geqslant|\tilde{Q} \cap \Omega| /|\tilde{Q}|$. Since $Q_{k}$ meets the complement of $\tilde{\Omega}$, we must have

$$
|Q \cap \Omega| \leqslant|\tilde{Q} \cap \Omega| \leqslant 2^{-n-1}|\tilde{Q}|=|Q| / 2,
$$

which clearly implies (b).

4. Estimates involving $f^{\#}$. The covering lemmas of the previous section are now applied to prove rearrangement function inequalities giving lower bounds for the sharp function. Inequality (1.1) is proved in Theorem 4.2 , making decisive use of Lemma 3.2. The first theorem proves an analog of (1.1) with $M f$ replaced by $f$.

4.1 THEOREM. There is a constant $C$ such that

$$
f_{w}^{*}(t) \leqslant C\left(f^{\sharp}\right)_{w}^{*}(2 t)+f_{w}^{*}(2 t)
$$

for $0<t<\infty$ and all locally integrable functions $f$.

Proof. Fix $t$. The set

$$
G=\left\{x: f^{\sharp}(x)>\left(f^{\sharp}\right)_{w}^{*}(2 t)\right\} \cup\left\{x:|f(x)|>f_{w}^{*}(2 t)\right\}
$$

has $w$-measure at most $4 t$. Let $\Omega$ be an open set containing $G$ with $w(\Omega)<5 t$. We claim that for sufficiently large $C$,

$$
w\left\{x \in \Omega:|f(x)|>C\left(f^{\#}\right)_{w}^{*}(2 t)+f_{w}^{*}(2 t)\right\} \leqslant t .
$$

We cover $\Omega$ by cubes as in Lemma 3.1 and consider any cube $Q$ in the covering. If $\left|f_{Q}\right|>f_{w}^{*}(2 t)$, then since $|f(x)| \leqslant f_{w}^{*}(2 t)$ on $\Omega^{\mathrm{c}}$ we must have

$$
\begin{aligned}
\int_{Q}\left|f(x)-f_{Q}\right| d x & \geqslant \int_{Q \cap \Omega^{c}}\left|f_{Q}-f(x)\right| d x \\
& \geqslant|| f_{Q}\left|-f_{w}^{*}(2 t)\right|\left|Q \cap \Omega^{c}\right| \geqslant|| f_{Q}\left|-f_{w}^{*}(2 t)\right||Q| / 2 .
\end{aligned}
$$

Since $f^{\#}(x) \leqslant\left(f^{\#}\right)_{w}^{*}(2 t)$ on $\Omega^{\mathrm{c}}$, we also have

$$
\int_{Q}\left|f(x)-f_{Q}\right| d x \leqslant|Q|\left(f^{\sharp}\right)_{w}^{*}(2 t)
$$

so that

$$
\left|f_{Q}\right| \leqslant 2\left(f^{\sharp}\right)_{w}^{*}(2 t)+f_{w}^{*}(2 t) .
$$

Set $\lambda=(1 / \varepsilon)\left(f^{\sharp}\right)_{w}^{*}(2 t)+\left|f_{Q}\right|$ and call $E=\{x \in Q:|f(x)|>\lambda\}$. Since we clearly have

$$
\left(\lambda-\left|f_{Q}\right|\right)|E| \leqslant \int_{Q}|| f(x)|-| f_{Q}|| d x \leqslant \int_{Q}\left|f(x)-f_{Q}\right| d x \leqslant|Q|\left(f^{\sharp}\right)_{w}^{*}(2 t),
$$


this implies $|E| \leqslant \varepsilon|Q|$. Choose $C=2+1 / \varepsilon$. Then

$$
\left|\left\{x \in Q:|f(x)|>C\left(f^{\sharp}\right)_{w}^{*}(2 t)+f_{w}^{*}(2 t)\right\}\right| \leqslant \varepsilon|Q| \text {. }
$$

By (2.3) we may choose $\varepsilon>0$ in such a way that $E \subset Q$ and $|E| \leqslant \varepsilon|Q|$ imply $w(E) \leqslant w(Q) / 5 B$. Since the same $\varepsilon$ works for all cubes in the covering, we obtain

$$
w\left\{x:|f(x)|>C\left(f^{\#}\right)_{w}^{*}(2 t)+f_{w}^{*}(2 t)\right\} \leqslant \frac{1}{5 B} \sum w\left(Q_{k}\right) \leqslant \frac{w(\Omega)}{5}<t .
$$

By the definition of weighted rearrangements, the proof is complete.

4.2 THEOREM. There is a constant $C$ such that

$$
(M f)_{w}^{*}(t) \leqslant C\left(f^{\sharp}\right)_{w}^{*}(2 t)+(M f)_{w}^{*}(2 t)
$$

for $0<t<\infty$ and all locally integrable functions $f$.

Proof. As above, we let $\Omega$ be an open set containing

$$
\left\{x: f^{\sharp}(x)>\left(f^{\sharp}\right)_{w}^{*}(2 t)\right\} \cup\left\{x: M f(x)>(M f)_{w}^{*}(2 t)\right\}
$$

and having $w(\Omega)<5 t$. We use Lemma 3.2 to cover $\Omega$. As in the proof of Theorem 4.1 , it suffices to find a $C$ such that

$$
\left|\left\{x \in Q: M f(x)>C\left(f^{\sharp}\right)_{w}^{*}(2 t)+(M f)_{w}^{*}(2 t)\right\}\right| \leqslant \varepsilon|Q|
$$

for all cubes $Q$ in the cover.

Let $Q$ be a cube in the cover and call $Q^{*}$ its triple. Fix $\lambda>(M f)_{w}^{*}(2 t)$, and let $E=\{x \in Q: M f(x)>\lambda\}$. For each $x \in E$ there is a cube $Q(x)$ with $x \in Q(x)$ and

$$
\frac{1}{|Q(x)|} \int_{Q(x)}|f(y)| d y>\lambda \text {. }
$$

We now invoke part (b) of our covering lemma. Since $M f>\lambda$ on $Q(x), Q(x) \subset \Omega$. Consequently, $|Q(x)|<|Q|$ and, hence, $Q(x) \subset Q^{*}$; this is the key observation. Since $Q^{*}$ meets $\Omega^{c},\left|f_{Q^{*}}\right| \leqslant(M f)_{w}^{*}(2 t)$. Accordingly,

$$
\left[\lambda-(M f)_{w}^{*}(2 t)\right]|Q(x)| \leqslant \int_{Q(x)}\left[|f(y)|-\left|f_{Q^{*}}\right|\right] d y \leqslant \int_{Q(x)}\left|f(y)-f_{Q^{*}}\right| d y .
$$

By the Vitali covering lemma, there are $x_{i} \in E$ such that the corresponding cubes $Q\left(x_{i}\right)$ are pairwise disjoint and satisfy $|E| \leqslant C_{n} \sum\left|Q\left(x_{i}\right)\right|$. Since $\cup Q\left(x_{i}\right) \subset Q^{*}$, we have

$$
\begin{aligned}
{\left[\lambda-(M f)_{w}^{*}(2 t)\right]|E| } & \leqslant C_{n} \int_{Q^{*}}\left|f(y)-f_{Q^{*}}\right| d y \\
& \leqslant C_{n}\left|Q^{*}\right|\left(f^{\sharp}\right)_{w}^{*}(2 t)=3^{n} C_{n}|Q|\left(f^{\sharp}\right)_{w}^{*}(2 t) .
\end{aligned}
$$

Choosing $\lambda=C\left(f^{\sharp}\right)_{w}^{*}(2 t)+(M f)_{w}^{*}(2 t)$ with $C=3^{n} C_{n} / \varepsilon$ gives

$$
\left|\left\{x \in Q: M f(x)>C\left(f^{\sharp}\right)_{w}^{*}(2 t)+(M f)_{w}^{*}(2 t)\right\}\right|=|E| \leqslant \varepsilon|Q|,
$$

and the proof is complete.

As in [1 and 3], the inequalities in 4.1 and 4.2 may be iterated to provide pointwise estimates for $f_{w}^{*}(t)$ and $(M f)_{w}^{*}(t)$. We state these below and omit their simple proofs. 
4.3 COROLLARY. For all locally integrable $f$,

(a)

$$
f_{w}^{*}(t) \leqslant C \int_{t}^{\infty}\left(f^{\#}\right)_{w}^{*}(s) \frac{d s}{s}+\lim _{r \rightarrow \infty} f_{w}^{*}(r)
$$

and

(b)

$$
(M f)_{w}^{*}(t) \leqslant C \int_{t}^{\infty}\left(f^{\sharp}\right)_{w}^{*}(s) \frac{d s}{s}+\lim _{r \rightarrow \infty}(M f)_{w}^{*}(r) .
$$

We note that $(b)$ gives the estimate

$$
\left(\int M f(x)^{p} w(x) d x\right)^{1 / p} \leqslant C p\left(\int f^{\sharp}(x)^{p} w(x) d x\right)^{1 / p}
$$

as long as $(M f)_{w}^{*}(t) \rightarrow 0$ as $t \rightarrow \infty$; this improves the bound obtained by Fefferman and Stein which grows exponentially in $p$ instead of linearly. While (a) gives a similar $L^{p}$-bound for $f$ instead of $M f$ and hence appears weaker, note that $f_{w}^{*}(t) \rightarrow 0$ does not imply $(M f)_{w}^{*}(t) \rightarrow 0$. Conversely, a weighted $L^{p}$-bound for $f$ does not imply a similar bound for $M f$ for all $p$, so (a) and (b) appear to be independent.

4.4 COROLlary. For locally integrable $f$,

(a)

$$
f_{w}^{* *}(t) \leqslant f_{w}^{*}(t)+C\left(f_{w}^{\#) * *}(t)\right.
$$

and

(b)

$$
(M f)_{w}^{* *}(t) \leqslant(M f)_{w}^{*}(t)+C\left(f^{\sharp}\right)_{w}^{* *}(t) .
$$

Inequalities of this form were introduced in Bennett, DeVore and Sharpley [2]; we gave a method for deriving such inequalities in [1]. Since these inequalities give local exponential integrability estimates in terms of the behavior of $f^{\#}$, they may be thought of as variants of the John-Nirenberg lemma.

\section{BIBLIOGRAPHY}

1. R. J. Bagby and D. S. Kurtz, $A$ rearranged good $\lambda$ inequality, preprint.

2. C. Bennett, R. DeVore and R. Sharpley, Weak- $L^{\infty}$ and BMO, Ann. of Math. (2) 113 (1981), 601-611.

3. C. Bennett and R. Sharpley, Weak-type inequalities for $H^{p}$ and BMO, Proc. Sympos. Pure Math., Vol. 35, Part 1, Amer. Math. Soc., Providence, R.I., 1979, pp. 201-229.

4. R. R. Coifman and C. Fefferman, Weighted norm inequalities for maximal functions and singular integrals, Studia Math. 51 (1974), 241-250.

5. C. Fefferman and E. M. Stein, $H^{p}$ spaces of several variables, Acta Math. 129 (1972), 137-193.

6. F. John and L. Nirenberg, On functions of bounded mean oscillation, Comm. Pure Appl. Math. 14 (1961), 415-426.

7. B. Muckenhoupt, Weighted inequalities for the Hardy maximal function, Trans. Amer. Math. Soc. 165 (1972), 207-226.

8. E. M. Stein and G. Weiss, Introduction to Fourier analysis on Euclidean spaces, Princeton Univ. Press, Princeton, N.J., 1971.

Department of Mathematical Sciences, New Mexico State University, las Cruces, New MEXICO 